\title{
Determining arbitrary Feynman integrals by vacuum integrals
}

\author{
Xiao Liu, ${ }^{1, *}$ and Yan-Qing $\mathrm{Ma}^{1,2,3, \dagger}$ \\ ${ }^{1}$ School of Physics and State Key Laboratory of Nuclear Physics and Technology, Peking University, \\ Beijing 100871, China \\ ${ }^{2}$ Center for High Energy Physics, Peking University, Beijing 100871, China \\ ${ }^{3}$ Collaborative Innovation Center of Quantum Matter, Beijing 100871, China
}

(Received 9 February 2018; published 11 April 2019)

\begin{abstract}
By introducing an auxiliary parameter, we find a new representation for Feynman integrals, which defines a Feynman integral by analytical continuation of a series containing only vacuum integrals. The new representation therefore conceptually translates the problem of computing Feynman integrals to the problem of performing analytical continuations. As an application of the new representation, we use it to construct a novel reduction method for multiloop Feynman integrals, which is expected to be more efficient than the known integration-by-parts reduction method. Using the new method, we successfully reduced all complicated two-loop integrals in the $g g \rightarrow H H$ process and $g g \rightarrow g g g$ process.
\end{abstract}

DOI: $10.1103 /$ PhysRevD.99.071501

\section{INTRODUCTION}

Computation of Feynman loop integrals is in the heart of modern physics, which is important both for testing the particle physics standard model and for discovering new physics. A good method to compute one-loop integrals was proposed as early as the 1970 s, the strategy of which is to first express scattering amplitudes in terms of linear combinations of master integrals (MIs) and then compute these MIs [1-3]. Based on this method, one can compute one-loop scattering amplitudes systematically and efficiently if the number of external legs is no more than 4. With further improvement of the traditional tensor reduction [4] and the development of unitarity-based reduction [5-7], computation of multileg one-loop scattering amplitudes is also a solved problem right now.

Yet, about 40 years later, it is still a challenge to compute multiloop integrals, even for two-loop integrals with four external legs. The mainstream approach to calculate multiloop integrals in the literature is similar to that at one-loop level, by first reducing Feynman integrals to MIs and then calculating these MIs. However, both of the two steps are much harder to achieve than the one-loop case.

Although compact and explicit expressions for one-loop MIs can be easily obtained [2,3], the computation of multiloop MIs is very challenging. There are many methods

\footnotetext{
xiao6@pku.edu.cn

yqma@pku.edu.cn
}

Published by the American Physical Society under the terms of the Creative Commons Attribution 4.0 International license. Further distribution of this work must maintain attribution to the author(s) and the published article's title, journal citation, and DOI. Funded by SCOAP. in the literature to compute multiloop MIs, such as the sector decomposition [8-19], Mellin-Barnes representation [19-34], and the differential equation method [35-40], but none of them provides a fully satisfactory solution. In Ref. [41], we proposed a systematic and efficient method to calculate multiloop MIs by constructing and numerically solving a system of ordinary differential equations (ODEs). The differential variable, say $\eta$, is an auxiliary parameter introduced to all Feynman propagators. With the ODEs, physical results at $\eta=0^{+}$are fully determined by boundary conditions chosen at $\eta=\infty$, which can be obtained almost trivially. Therefore, MIs can be treated as special functions in our method, but it relies on a good reduction method to set up ODEs.

Reduction of multiloop integrals is an even harder problem. Significantly different from the one-loop case, propagators in a multiloop integral are usually not enough to form a complete set to expand all independent scalar products, either between a loop momentum and an external momentum or between two loop momenta. As a consequence, the unitaritybased multiloop reduction [42-52] has difficulty fully reducing scattering amplitudes. Although the integrationby-parts (IBP) reduction [53-57] is general enough to reduce any scattering amplitude to MIs, the incompleteness of multiloop propagators makes it hard to generate efficient reduction relations. Currently, IBP reduction is mainly based on Laporta's algorithm [54], which usually generates huge number of linear equations, which is extremely hard to solve for multiscale problems. For example, it cannot give a complete reduction for Higgs pair hadroproduction at twoloop order [58]. Improvements for IBP reduction method can be found in $[59,60]$ and references therein.

Inspired by our previous work [41], in this article we construct a novel method to compute Feynman loop 
integrals. The key observation is that, after introducing the auxiliary parameter $\eta$, any Feynman integral can be defined as the analytical continuation of a calculable asymptotic series, which contains only simple vacuum integrals. The series can thus be thought of as a new representation of the Feynman integral. Based on the new representation, we construct an efficient reduction method for multiloop integrals. We demonstrate the correctness and efficiency of our reduction method with two cutting-edge examples.

\section{A NEW REPRESENTATION FOR FEYNMAN INTEGRALS}

Following Ref. [41], we introduce a dimensionally regularized $L$-loop Feynman integral with an auxiliary parameter $\eta$,

$$
\mathcal{M}(D, \vec{s}, \eta) \equiv \int \prod_{i=1}^{L} \frac{\mathrm{d}^{D} \ell_{i}}{\mathrm{i} \pi^{D / 2}} \prod_{\alpha=1}^{N} \frac{1}{\left(\mathcal{D}_{\alpha}+\mathrm{i} \eta\right)^{\nu_{\alpha}}},
$$

where $D$ is the spacetime dimension, $\mathcal{D}_{\alpha} \equiv q_{\alpha}^{2}-m_{\alpha}^{2}$ are usual inverse Feynman propagators with $m_{\alpha}$ being corresponding masses and $q_{\alpha}$ being linear combinations of loop momenta $\ell_{i}$ and external momenta $p_{i}, \vec{s}=\left(s_{1}, \ldots, s_{r}\right)$ are independent kinematic variables (including mass parameters) in the problem, and $\nu_{\alpha}$ are powers of propagators whose dependence is suppressed in the left-hand side of the equation. The physical integral that we want to get is

$$
\mathcal{M}\left(D, \vec{s}, 0^{+}\right) \equiv \lim _{\eta \rightarrow 0^{+}} \mathcal{M}(D, \vec{s}, \eta),
$$

with $0^{+}$defining the causality of Feynman amplitudes.

The study in Ref. [41] shows that, as $\eta \rightarrow \infty$, there is only one integration region for $\mathcal{M}(D, \vec{s}, \eta)$, where all components of loop momenta are of the order of $|\eta|^{1 / 2}$. Therefore, all propagators can be expanded like

$$
\begin{aligned}
& \frac{1}{\left[(\ell+p)^{2}-m^{2}+\mathrm{i} \eta\right]^{\nu}} \\
& =\frac{1}{\left(\ell^{2}+\mathrm{i} \eta\right)^{\nu}} \sum_{j=0}^{\infty} \frac{(\nu)_{j}}{j !}\left(-\frac{2 \ell \cdot p+p^{2}-m^{2}}{\ell^{2}+\mathrm{i} \eta}\right)^{j},
\end{aligned}
$$

where $\ell$ is a linear combination of loop momenta $\ell_{i}, p$ is a linear combination of external momenta $p_{i}$, and $(\nu)_{j} \equiv$ $\nu(\nu+1) \cdots(\nu+j-1)$ is the Pochhammer symbol. After the expansion, all external momenta and masses are not present in denominators anymore; thus each term of the expansion can be interpreted as vacuum integrals with equal internal squared masses -i $\eta$. Inserting Eq. (3) into Eq. (1) and rescaling all loop momenta by $\eta^{1 / 2}$, we can obtain an asymptotic expansion around $\eta=\infty$,

$$
\mathcal{M}(D, \vec{s}, \eta)=\eta^{L D / 2-\sum_{\alpha} \nu_{\alpha}} \sum_{\mu_{0}=0}^{\infty} \eta^{-\mu_{0}} \mathcal{M}_{\mu_{0}}^{\mathrm{bub}}(D, \vec{s}),
$$

where the superscript "bub" means vacuum bubble integrals. Although asymptotic expansion of Feynman integrals itself is not new [61,62], the novelty here is that our expansion is with respect to an auxiliary parameter introduced by hand and thus can be applied to any problem. In Eq. (4), $\mathcal{M}_{\mu_{0}}^{\mathrm{bub}}(D, \vec{s})$ consist of vacuum integrals with equal internal squared masses $-\mathrm{i}$, which can be easily reduced to vacuum MIs denoted by $\left\{I_{L, 1}^{\mathrm{bub}}(D), \ldots, I_{L, B_{L}}^{\mathrm{bub}}(D)\right\}$. Here $B_{L}$ is the total number of $L$-loop equal-mass vacuum MIs, with $B_{1}=1, B_{2}=2, B_{3}=5$ and so on. Thus, after the vacuum reduction, we have the decomposition

$\mathcal{M}_{\mu_{0}}^{\mathrm{bub}}(D, \vec{s})=\sum_{k=1}^{B_{L}} I_{L, k}^{\mathrm{bub}}(D) \sum_{\vec{\mu} \in \Omega_{\mu_{0}}^{r}} C_{k}^{\mu_{0} \ldots \mu_{r}}(D) s_{1}^{\mu_{1}} \cdots s_{r}^{\mu_{r}}$,

where $\vec{\mu}$ is a $r$-dimensional vector in $\Omega_{\mu_{0}}^{r} \equiv$ $\left\{\vec{\mu} \in \mathbb{N}^{r} \mid \mu_{1}+\cdots+\mu_{r}=\mu_{0}\right\}$, and $C_{k}^{\mu_{0} \ldots \mu_{r}}(D)$ are rational functions of $D$.

As vacuum MIs can be easily calculated [63-68], the series (4) defines an analytical function around $\eta=\infty$, which therefore determines $\mathcal{M}(D, \vec{s}, \eta)$ for any value of $\eta$ based on analytical continuation. Especially, the desired physical value at $\eta=0^{+}$is fully determined. As a result, the expression (4) can be thought as a new series representation of $\mathcal{M}\left(D, \vec{s}, 0^{+}\right)$. Then the problem of computing Feynman integrals is translated to the problem of performing analytical continuations. This conceptual change of interpretation of Feynman integrals may both deepen our understanding of scattering amplitudes and result in powerful methods to compute scattering amplitudes.

Beginning in the next section, we are devoted to constructing a powerful reduction method for Feynman integrals based on the new representation. The reduction method can not only reduce any Feynman integral to MIs $\vec{I}(D, \vec{s}, \eta)$ (note that there are more MIs after the introduction of $\eta$ ), but also set up a system of ODEs for these MIs,

$$
\frac{\partial}{\partial \eta} \vec{I}(D, \vec{s}, \eta)=A(D, \vec{s}, \eta) \vec{I}(D, \vec{s}, \eta) .
$$

Then the analytical continuation from $\eta=\infty$ to $\eta=0^{+}$can be realized by solving the ODEs [41].

\section{REDUCTION RELATIONS FROM THE NEW REPRESENTATION}

An important property of Feynman loop integrals is that the number of MIs is finite [69]. More precisely, for loop integrals constructed from any given set of propagators, there exists a finite set of loop integrals called MIs so that 
all other loop integrals can be expressed as linear combinations of them, with coefficients being rational functions of kinematic variables and spacetime dimension. The reduction is to find relations among loop integrals and eventually express all loop integrals by MIs.

Let us first study how to find relations among a given set of loop integrals using the new representation. Suppose we have a set of integrals $G=\left\{\mathcal{M}_{1}, \ldots, \mathcal{M}_{n}\right\}$. Linear relations among them can be written as

$$
\sum_{i=1}^{n} Q_{i}(D, \vec{s}, \eta) \mathcal{M}_{i}(D, \vec{s}, \eta)=0,
$$

where $Q_{i}$ are homogeneous polynomials of $\eta$ and kinematic variables $\vec{s}$. We denote the mass dimension of $\mathcal{M}_{i}$ by $\operatorname{Dim}\left(\mathcal{M}_{i}\right)$ and the degree of $Q_{i}$ by $d_{i}$, which are constrained by

$$
2 d_{1}+\operatorname{Dim}\left(\mathcal{M}_{1}\right)=\cdots=2 d_{n}+\operatorname{Dim}\left(\mathcal{M}_{n}\right) .
$$

Therefore, there is only one degree of freedom in $\left\{d_{i}\right\}$, which can be chosen as $d_{\max }=\max \left\{d_{i}\right\}$.

For any given $d_{\max } \geq 0$, we can expand each $Q_{i}(D, \vec{s}, \eta)$ as

$$
Q_{i}(D, \vec{s}, \eta)=\sum_{\left(\lambda_{0}, \vec{\lambda}\right) \in \Omega_{d_{i}}^{r+1}} Q_{i}^{\lambda_{0} \cdots \lambda_{r}}(D) \eta^{\lambda_{0}} s_{1}^{\lambda_{1}} \cdots s_{r}^{\lambda_{r}}
$$

where $Q_{i}^{\lambda_{0} \ldots \lambda_{r}}(D)$ are rational functions of $D$ to be determined [note that by definition $Q_{i}(D, \vec{s}, \eta) \equiv 0$ if $\left.d_{i}<0\right]$. As the series (4) fully determines all analytical functions $\mathcal{M}_{i}$, it certainly also determines the relations among them in Eq. (7). To determine the unknown coefficients $Q_{i}^{\lambda_{0} \ldots \lambda_{r}}(D)$, we substitute Eqs. (4), (5), and (9) into Eq. (7) and then expand it in terms of monomials of $I_{L, k}^{\mathrm{bub}}(D), \eta$, and $\vec{s}$, which gives

$$
\sum_{k, \rho_{0}, \vec{\rho}} f_{k}^{\rho_{0} \ldots \rho_{r}} I_{L, k}^{\mathrm{bub}}(D) \eta^{\rho_{0}} s_{1}^{\rho_{1}} \ldots s_{r}^{\rho_{r}}=0,
$$

where $f_{k}^{\rho_{0} \ldots \rho_{r}}$ are linear functions of $Q_{i}^{\lambda_{0} \ldots \lambda_{r}}(D)$. As $I_{L, k}^{\mathrm{bub}}(D) \eta^{\rho_{0}} s_{1}^{\rho_{1}} \ldots s_{r}^{\rho_{r}}$ are independent of each other, their coefficients must vanish, which results in a system of linear equations

$$
f_{k}^{\rho_{0} \ldots \rho_{r}}=0, \quad \text { for each } k, \rho_{0}, \ldots, \rho_{r} .
$$

By calculating the series (4) to sufficiently high order in $1 / \eta$, we can generate enough linear equations to constrain the solution space of $Q_{i}^{\lambda_{0} \ldots \lambda_{r}}(D)$. In practice, we find that it is sufficient if the number of linear equations is larger than the number of unknown coefficients $Q_{i}^{\lambda_{0} \ldots \lambda_{r}}(D)$ by $30 \%$.
Once the solution space is obtained, it provides us with all relations among the integral set $G$ with given $d_{\max }$.

Now let us consider two sets of integrals, $G_{1}$ and $G_{2}$, with the condition that integrals in $G_{2}$ are all simpler than integrals in $G_{1}$. Assuming that $G_{1}$ can be reduced to $G_{2}$, we provide an algorithm to find out relations to realize this reduction.

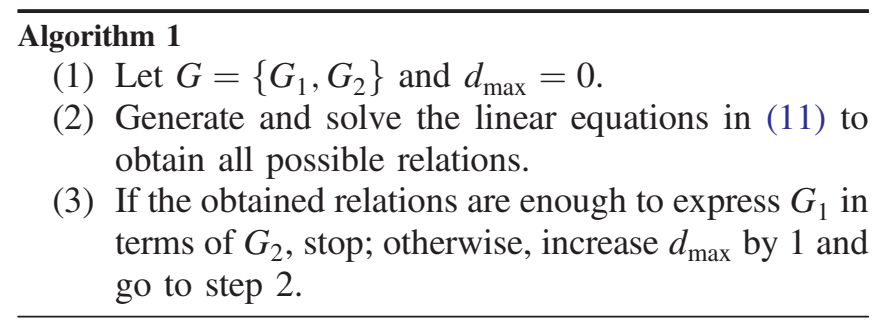

According to our assumption, the iteration must terminate after finitely many steps, because $Q_{i}(D, \vec{s}, \eta)$ are polynomials with finite degree. Our algorithm is constructed to search for as simple as possible relations.

We emphasize that, although they are determined by the region $\eta \rightarrow \infty$, the obtained relations are correct for any value of $\eta$ and thus can be used to reduce physical Feynman loop integrals.

\section{REDUCTION SCHEME}

To reduce a given integral to simpler integrals, we still need to choose $G_{1}$, which includes the given integral as an element, and $G_{2}$, which includes only simpler integrals and can express all integrals in $G_{1}$. There are many possible choices, but a good choice should satisfy the following: (1) Relations among $\left\{G_{1}, G_{2}\right\}$ are simple, so that they can be easily found using algorithm 1; (2) the number of integrals in $G_{1}$ is not too large, so that one can efficiently solve the obtained relations to reduce $G_{1}$. To simplify our discussion, in the following we only consider the reduction of scalar integrals, ${ }^{2}$ which means integrals with no numerator in the integrand.

Let us begin with introducing some notations. For a given set of propagators, a scalar integral can be denoted by its powers of corresponding propagators $\vec{\nu}=\left(\nu_{1}, \ldots, \nu_{N}\right)$ $\left(\nu_{i} \geq 0\right)$. A sector is a set of integrals that have exactly the same 0 s in the powers; e.g., $(5,1,0,3)$ and $(7,8,0,9)$ are in the same sector. We introduce two sets of operators $\mathbf{m}^{+}$ and $\mathbf{m}^{-}$, with positive integer $m$. If $m>1$, we define

\footnotetext{
${ }^{1}$ In practice, we solve the solution space with some chosen nonspecial values of $D$, e.g., $D=1867 / 5281$, and then reconstruct the $D$-dependence for the solutions. Finite field technique $[59,70]$ is also used to speed up the calculation.

${ }^{2}$ Note that reduction of scalar integrals is already general enough. First, any tensor integral can be easily expressed as scalar integrals in a higher spacetime dimension [71-73]. Second, recurrence relations to relate higher spacetime-dimension integrals to lower spacetime-dimension integrals [72,74] can be obtained by reduction relations of scalar integrals.
} 
$\mathbf{m}^{ \pm}=(\mathbf{m}-\mathbf{1})^{ \pm} \mathbf{1}^{ \pm} \cdot \mathbf{1}^{+}\left(\mathbf{1}^{-}\right)$is defined so that, when applying it on an integral $\vec{\nu}$, it generates all integrals with one nonvanishing $\nu_{i}$ increased (decreased) by 1 . For example, $\mathbf{1}^{+}(5,1,0,3)=\{(6,1,0,3),(5,2,0,3),(5,1,0,4)\}$ generating integrals in the same sector, and $\mathbf{1}^{-}(5,1,0,3)=$ $\{(4,1,0,3),(5,0,0,3),(5,1,0,2)\}$ generating integrals either in the same sector or in subsectors. Note that $\mathbf{m}^{+} \mathbf{n}^{-} \neq \mathbf{n}^{-} \mathbf{m}^{+}$, which can be easily verified.

To figure out a good choice of $\left\{G_{1}, G_{2}\right\}$ at multiloop level, we should first take a look at the one-loop case to see what we can learn. In this case, it is well known that quite simple relations can be obtained to reduce integrals $G_{1}=$ $\mathbf{1}^{+} \vec{\nu}$ to simpler integrals $G_{2}=\mathbf{1}^{-} \mathbf{1}^{+} \vec{\nu}$ [75]. This reduction is possible because there is only one MI in each sector at oneloop level, and thus even integrals like $\mathbf{1}^{+}(1,1,0,1)$ are fully reducible.

As a natural generalization of one-loop strategy, we propose to reduce integrals $G_{1}=\mathbf{m}^{+} \vec{\nu}$ at multiloop level, where $m$ is usually larger than 1 because there is usually more than one MI in each sector. The smallest allowed value of $m$, which guarantees that all integrals $\mathbf{m}^{+} \vec{\nu}$ are reducible to simpler integrals, can be found out within our method. Alternatively, it can be easily determined by investigating the distribution of MIs in the sector containing $\vec{\nu}$, because finding out MIs is a simple problem [76]. In the following examples, we find $m=2$ or 3; thus there are only dozens of integrals in $G_{1}$.

A possible generalization for the set of simpler integrals is then $G_{2}=\left\{\mathbf{1}^{-} \mathbf{m}^{+}, \mathbf{2}^{-} \mathbf{m}^{+}, \ldots, \mathbf{m}^{-} \mathbf{m}^{+}\right\} \vec{\nu}$, or its subset $G_{2}=\left\{\mathbf{1}^{-} \mathbf{m}^{+}, \mathbf{1}^{-}(\mathbf{m}-1)^{+}, \ldots, \mathbf{1}^{-} \mathbf{1}^{+}\right\} \vec{\nu}$. In the following examples, we use the latter choice and find that it can already result in not too complicated relations.

There are exceptions where a reducible integral cannot be included in any fully reducible set $\mathbf{m}^{+} \vec{\nu}$. This is harmless because it only happens when this integral and some MIs have the same $|\vec{\nu}|$. We can either put it in a partially reducible set, or simply treat it as a redundant MI.

With the above strategy, we can express any reducible integral as linear combinations of simpler integrals. Then by iteration, we can reduce any integral to MIs.

Therefore, we realize a step-by-step reduction scheme for multiloop integrals, which is similar to the one-loop case. Comparing with the traditional IBP reduction method, an advantage of our method is that we never encounter large coupled linear systems. As a result, the computation complexity of numerically solving the obtained reduction relations to reduce $N$ integrals to MIs is $O(N)$, rather than $O\left(N^{3}\right)$ in the fully coupled case.

\section{EXAMPLES}

To test the power of our new reduction method, we apply it to two cutting-edge processes. The first example is a twoloop four-scale on-shell scattering $g g \rightarrow H H$ with a top quark loop. The second one is two-loop five-gluon on-shell scattering process with five independent scales. Integrals of

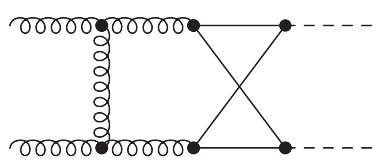

(a)

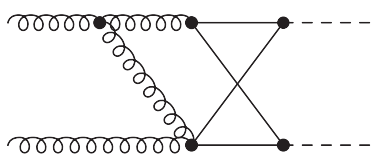

(c)

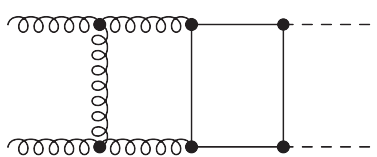

(b)

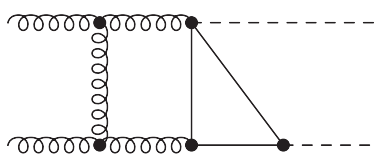

(d)
FIG. 1. Some typical diagrams in the $g g \rightarrow H H$ process. Diagrams (c) and (d) are obtained from (a) and (b) by shrinking a gluon and a top quark line, respectively.

the two examples have not been fully reduced by the traditional IBP reduction method [58].

We have tried integrals in many sectors, and found all of them can be easily reduced using our method. Three conclusions based on our test are as follows. First, as expected the more external legs the harder the reduction is. Second, reduction for nonplanar integrals is typically harder than planar integrals, which may be caused by the fact that there are usually more MIs in nonplanar sectors. Finally, suppose that $\vec{v}$ and $\vec{e}$ belong to the same sector and that $\vec{e}$ is the simplest integral, which includes only single power propagators; then the reduction for $\mathbf{m}^{+} \vec{e}$ is usually more difficult than the first-step reduction for $\mathbf{m}^{+} \vec{\nu}$. This can be understood because the set $\left\{\mathbf{1}^{-} \mathbf{m}^{+}, \ldots, \mathbf{1}^{-} \mathbf{1}^{+}\right\} \vec{e}$ contains fewer integrals in the leading sector and thus has fewer flexible relations to express desired reducible integrals. With these observations, we then mainly discuss potentially difficult integrals.

For the first example, some typical diagrams are shown in Fig. 1, and corresponding reduction information is summarized in Table I. Here, in addition to two potentially difficult nonplanar diagrams, we also show two planar diagrams to compare with. We find that the six-propagator nonplanar sector Fig. 1(c) is most difficult to reduce among all sectors in this process. To reduce $\mathbf{3}^{+} \vec{e}$ in this sector, which contains 56 integrals, we need to set up 55 relations with $d_{\max }=1$ and 1 relation with $d_{\max }=5$.

For the second example, we show all nonplanar five-leg topologies in Fig. 2, and summarize corresponding reduction information in Table I. Among them, the most

TABLE I. Main reduction information for sectors shown in Figs. 1 and 2. See text for details.

\begin{tabular}{|c|c|c|c|c|c|c|c|}
\hline \multicolumn{4}{|c|}{$g+g \rightarrow H+H$} & \multicolumn{4}{|c|}{$g+g \rightarrow g+g+g$} \\
\hline Sector & Type & $d_{\max }$ & $\mathbf{m}^{+}$ & Sector & Type & $d_{\max }$ & $\mathbf{m}^{+}$ \\
\hline 1(a) & 7-NP & 1 & $3^{+}$ & 2(a) & 8-NP & 1 & $3^{+}$ \\
\hline 1(b) & 7-P & 1 & $3^{+}$ & 2(b) & 8-NP & 3 & $3^{+}$ \\
\hline $1(\mathrm{c})$ & 6-NP & 5 & $3^{+}$ & $2(\mathrm{c})$ & $7-\mathrm{NP}$ & 4 & $3^{+}$ \\
\hline 1(d) & $6-\mathrm{P}$ & 4 & $\mathbf{2}^{+}$ & $2(d)$ & 6-NP & 2 & $\mathbf{3}^{+}$ \\
\hline
\end{tabular}




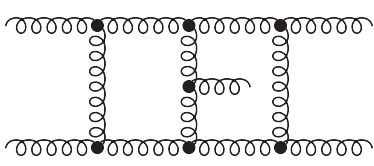

(a)

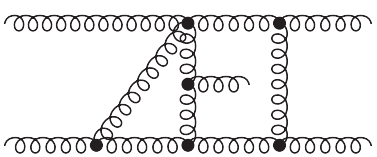

(c)

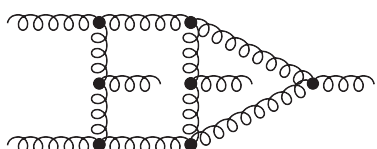

(b)

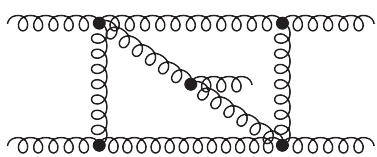

(d)
FIG. 2. All nonplanar five-leg diagrams for the five-gluon process.

complicated one is the seven-propagator sector represented by Fig. 2(c). To reduce $3^{+} \vec{e}$ in this sector, which contains 84 integrals, we need to set up 14 relations with $d_{\max }=0,64$ relations with $d_{\max }=1,4$ relations with $d_{\max }=2$, and 2 relations with $d_{\max }=4$.

Reduction relations for $\mathbf{m}^{+} \vec{e}$ of all sectors listed in Table I were obtained on a laptop with 4 core Intel i76500U CPU and 16 GB of RAM within 1 day, and final analytical relations are available for download in electronic form from an ancillary file in the arXiv version. For a given phase space point, a given spacetime dimension $D$, and assuming that values of all simpler integrals are already known, then solving all these $\mathbf{m}^{+} \vec{e}$ by Gaussian elimination of obtained relations can be finished within 0.01 second, which should be efficient enough to do phase space integration. To compare with, FIRE5 [57] needs several hours to reduce $\mathbf{m}^{+} \vec{e}$ to MIs at a given phase space point. Though the final results obtained by FIRE5 are analytic in spacetime dimension $D$, we expect that it will not reach as high an efficiency as ours even if it works with a specific value of $D$. We have checked point by point in phase space that relations obtained by our method agree with that obtained by FIRE5. Technical details will be given in a forthcoming paper [77].

\section{SUMMARY AND OUTLOOK}

In this article, we propose a new representation for Feynman integrals, which is defined by analytical continuation of an asymptotic series containing only vacuum integrals. The new representation translates the problem of computing Feynman integrals to the problem of performing analytical continuations. This new perspective of Feynman integrals may be helpful to deepen our understanding of Feynman integrals and scattering amplitudes.

As an application of the new representation, we construct a systematic and efficient reduction method for multiloop Feynman integrals. Different from the traditional IBP reduction method based Laporta's algorithm, we never involve large coupled linear systems because our method reduces integrals step by step, similar to the one-loop case. Therefore, once reduction relations in our method are obtained, the numerical evaluation can be much more efficient than IBP reduction, especially when dealing with multiscale problems. With two two-loop cutting-edge examples, we find that our method is indeed very powerful to reduce multiloop multiscale Feynman integrals.

In our reduction method, the appearance of additional masses does not introduce too many difficulties, because we have already introduced effective masses for each propagator. Therefore, for instance, the reduction of twoloop integrals in the five-gluon scattering process with a massive quark loop and $g g \rightarrow t \bar{t} g$ process, which are exceedingly difficult problems in the view of IBP reduction, should be achievable based on our method.

\section{ACKNOWLEDGMENTS}

We thank Kuang-Ta Chao, Feng Feng, Yu Jia, David Kosower, Roman Lee, Zhao Li, Xiaohui Liu, Ce Meng, Jian-Wei Qiu, Chen-Yu Wang and Yang Zhang for helpful discussions. This work is in part supported by the Recruitment Program of Global Youth Experts of China.

Note added.-Recently, several preprints appeared, e.g., [78-94], which are aimed at solving two-loop cutting-edge problems with IBP reduction method but equipped with many advanced techniques. Even with these improvements of IBP reductions, our reduction method is still very competitive.
[1] G. Passarino and M. J. G. Veltman, One loop corrections for $e^{+} e^{-}$annihilation Into $\mu^{+} \mu^{-}$in the Weinberg model, Nucl. Phys. B160, 151 (1979).

[2] G. 't Hooft and M. J. G. Veltman, Scalar one loop integrals, Nucl. Phys. B153, 365 (1979).

[3] G. J. van Oldenborgh and J. A. M. Vermaseren, New algorithms for one loop integrals, Z. Phys. C 46, 425 (1990).
[4] A. Denner and S. Dittmaier, Reduction schemes for oneloop tensor integrals, Nucl. Phys. B734, 62 (2006).

[5] R. Britto, F. Cachazo, and B. Feng, Generalized unitarity and one-loop amplitudes in $N=4$ super-Yang-Mills, Nucl. Phys. B725, 275 (2005).

[6] G. Ossola, C. G. Papadopoulos, and R. Pittau, Reducing full one-loop amplitudes to scalar integrals at the integrand level, Nucl. Phys. B763, 147 (2007). 
[7] W. T. Giele, Z. Kunszt, and K. Melnikov, Full one-loop amplitudes from tree amplitudes, J. High Energy Phys. 04 (2008) 049.

[8] T. Binoth and G. Heinrich, An automatized algorithm to compute infrared divergent multiloop integrals, Nucl. Phys. B585, 741 (2000).

[9] G. Heinrich,Sector decomposition, Int. J. Mod. Phys. A 23, 1457 (2008).

[10] S. Borowka, G. Heinrich, S. Jahn, S. P. Jones, M. Kerner, J. Schlenk, and T. Zirke, pySecDec: A toolbox for the numerical evaluation of multiscale integrals, Comput. Phys. Commun. 222, 313 (2018).

[11] J. Carter and G. Heinrich, SecDec: A general program for sector decomposition, Comput. Phys. Commun. 182, 1566 (2011).

[12] S. Borowka, J. Carter, and G. Heinrich, Numerical evaluation of multiloop integrals for arbitrary kinematics with SecDec 2.0, Comput. Phys. Commun. 184, 396 (2013).

[13] S. Borowka, G. Heinrich, S. P. Jones, M. Kerner, J. Schlenk, and T. Zirke, SecDec-3.0: Numerical evaluation of multiscale integrals beyond one loop, Comput. Phys. Commun. 196, 470 (2015).

[14] C. Bogner and S. Weinzierl, Resolution of singularities for multiloop integrals, Comput. Phys. Commun. 178, 596 (2008).

[15] A. V. Smirnov and M. N. Tentyukov, Feynman integral evaluation by a sector decomposiTion approach (FIESTA), Comput. Phys. Commun. 180, 735 (2009).

[16] A. V. Smirnov, V. A. Smirnov, and M. Tentyukov, FIESTA 2: Parallelizeable multiloop numerical calculations, Comput. Phys. Commun. 182, 790 (2011).

[17] A. V. Smirnov, FIESTA 3: Cluster-parallelizable multiloop numerical calculations in physical regions, Comput. Phys. Commun. 185, 2090 (2014).

[18] A. V. Smirnov, FIESTA4: Optimized Feynman integral calculations with GPU support, Comput. Phys. Commun. 204, 189 (2016).

[19] J. Gluza, K. Kajda, T. Riemann, and V. Yundin, Numerical evaluation of tensor Feynman integrals in Euclidean kinematics, Eur. Phys. J. C 71, 1516 (2011).

[20] N. I. Usyukina, On a representation for three point function, Teor. Mat. Fiz. 22, 300 (1975).

[21] V. A. Smirnov, Analytical result for dimensionally regularized massless on shell double box, Phys. Lett. B 460, 397 (1999).

[22] J. B. Tausk, Nonplanar massless two loop Feynman diagrams with four on-shell legs, Phys. Lett. B 469, 225 (1999).

[23] G. Heinrich and V. A. Smirnov, Analytical evaluation of dimensionally regularized massive on-shell double boxes, Phys. Lett. B 598, 55 (2004).

[24] M. Czakon, J. Gluza, and T. Riemann, Master integrals for massive two-loop bhabha scattering in QED, Phys. Rev. D 71, 073009 (2005).

[25] M. Czakon, Automatized analytic continuation of Mellin-Barnes integrals, Comput. Phys. Commun. 175, 559 (2006).

[26] C. Anastasiou and A. Daleo, Numerical evaluation of loop integrals, J. High Energy Phys. 10 (2006) 031.

[27] J. Gluza, K. Kajda, and T. Riemann, AMBRE: A mathematica package for the construction of Mellin-Barnes representations for Feynman integrals, Comput. Phys. Commun. 177, 879 (2007).

[28] I. Dubovyk, J. Gluza, and T. Riemann, nonplanar Feynman diagrams and Mellin-Barnes representations with AMBRE 3.0, J. Phys. Conf. Ser. 608, 012070 (2015).

[29] I. Dubovyk, J. Gluza, T. Riemann, and J. Usovitsch, Numerical integration of massive two-loop Mellin-Barnes integrals in Minkowskian regions, Proc. Sci., LL2016 (2016) 034.

[30] I. Dubovyk, A. Freitas, J. Gluza, T. Riemann, and J. Usovitsch, The two-loop electroweak bosonic corrections to $\sin ^{2} \theta_{\text {eff }}^{b}$, Phys. Lett. B 762, 184 (2016).

[31] J. Gluza, T. Jelinski, and D. A. Kosower, Efficient evaluation of massive Mellin-Barnes integrals, Phys. Rev. D 95, 076016 (2017).

[32] I. Dubovyk, J. Gluza, T. Jelinski, T. Riemann, and J. Usovitsch, New prospects for the numerical calculation of Mellin-Barnes integrals in Minkowskian kinematics, Acta Phys. Pol. B 48, 995 (2017).

[33] A. V. Sidorov, V. I. Lashkevich, and O. P. Solovtsova, Asymptotics of the contour of the stationary phase and efficient evaluation of the Mellin-Barnes integral for the $F_{3}$ structure function, Phys. Rev. D 97, 076009 (2018).

[34] M. Prausa, Mellin-Barnes meets method of brackets: A novel approach to Mellin-Barnes representations of Feynman integrals, Eur. Phys. J. C 77, 594 (2017).

[35] A. V. Kotikov, Differential equations method: New technique for massive Feynman diagrams calculation, Phys. Lett. B 254, 158 (1991).

[36] Z. Bern, L. J. Dixon, and D. A. Kosower, Dimensionally regulated one loop integrals, Phys. Lett. B 302, 299 (1993); Erratum, Phys. Lett. B 318, 649(E) (1993).

[37] E. Remiddi, Differential equations for Feynman graph amplitudes, Nuovo Cimento A 110, 1435 (1997).

[38] T. Gehrmann and E. Remiddi, Differential equations for two loop four point functions, Nucl. Phys. B580, 485 (2000).

[39] J. M. Henn, Multiloop integrals in dimensional regularization made simple, Phys. Rev. Lett. 110, 251601 (2013).

[40] R. N. Lee, Reducing differential equations for multiloop master integrals, J. High Energy Phys. 04 (2015) 108.

[41] X. Liu, Y.-Q. Ma, and C.-Y. Wang, A systematic and efficient method to compute multiloop master integrals, Phys. Lett. B 779, 353 (2018).

[42] J. Gluza, K. Kajda, and D. A. Kosower, Towards a basis for planar two-loop integrals, Phys. Rev. D 83, 045012 (2011).

[43] D. A. Kosower and K. J. Larsen, Maximal unitarity at two loops, Phys. Rev. D 85, 045017 (2012).

[44] P. Mastrolia, E. Mirabella, G. Ossola, and T. Peraro, Integrand-reduction for two-loop scattering amplitudes through multivariate polynomial division, Phys. Rev. D 87, 085026 (2013).

[45] S. Badger, H. Frellesvig, and Y. Zhang, Hepta-cuts of twoloop scattering amplitudes, J. High Energy Phys. 04 (2012) 055.

[46] P. Mastrolia, E. Mirabella, G. Ossola, and T. Peraro, Multiloop integrand reduction for dimensionally regulated amplitudes, Phys. Lett. B 727, 532 (2013).

[47] K. J. Larsen and Y. Zhang, Integration-by-parts reductions from unitarity cuts and algebraic geometry, Phys. Rev. D 93, 041701 (2016). 
[48] H. Ita, Two-loop integrand decomposition into master integrals and surface terms, Phys. Rev. D 94, 116015 (2016).

[49] S. Badger, G. Mogull, and T. Peraro, Local integrands for two-loop all-plus Yang-Mills amplitudes, J. High Energy Phys. 08 (2016) 063.

[50] P. Mastrolia, T. Peraro, and A. Primo, Adaptive integrand decomposition in parallel and orthogonal space, J. High Energy Phys. 08 (2016) 164.

[51] S. Abreu, F. F. Cordero, H. Ita, M. Jaquier, and B. Page, Subleading poles in the numerical unitarity method at two loops, Phys. Rev. D 95, 096011 (2017).

[52] S. Abreu, F. F. Cordero, H. Ita, M. Jaquier, B. Page, and M. Zeng, Two-Loop Four-Gluon Amplitudes from Numerical Unitarity, Phys. Rev. Lett. 119, 142001 (2017).

[53] K. G. Chetyrkin and F. V. Tkachov, Integration by parts: The algorithm to calculate beta functions in 4 loops, Nucl. Phys. B192, 159 (1981).

[54] S. Laporta, High precision calculation of multiloop Feynman integrals by difference equations, Int. J. Mod. Phys. A 15, 5087 (2000).

[55] C. Studerus, Reduze-Feynman integral reduction in $\mathrm{C}++$, Comput. Phys. Commun. 181, 1293 (2010).

[56] R. N. Lee, Presenting LiteRed: A tool for the Loop InTEgrals REDuction, arXiv:1212.2685.

[57] A. V. Smirnov, FIRE5: A C++ implementation of Feynman Integral REduction, Comput. Phys. Commun. 189, 182 (2015).

[58] S. Borowka, N. Greiner, G. Heinrich, S. Jones, M. Kerner, J. Schlenk, U. Schubert, and T. Zirke, Higgs Boson Pair Production in Gluon Fusion at Next-to-Leading Order with Full Top-Quark Mass Dependence, Phys. Rev. Lett. 117, 012001 (2016); Erratum, Phys. Rev. Lett. 117, 079901(E) (2016).

[59] A. von Manteuffel and R. M. Schabinger, A novel approach to integration by parts reduction, Phys. Lett. B 744, 101 (2015).

[60] J. Boehm, A. Georgoudis, K. J. Larsen, M. Schulze, and Y. Zhang, Complete sets of logarithmic vector fields for integration-by-parts identities of Feynman integrals, Phys. Rev. D 98, 025023 (2018)

[61] V. A. Smirnov, Asymptotic expansions in momenta and masses and calculation of Feynman diagrams, Mod. Phys. Lett. A 10, 1485 (1995).

[62] V. A. Smirnov, Applied asymptotic expansions in momenta and masses, Springer Tracts Mod. Phys. 177, 1 (2002).

[63] A. I. Davydychev and J. B. Tausk, Two loop selfenergy diagrams with different masses and the momentum expansion, Nucl. Phys. B397, 123 (1993).

[64] D. J. Broadhurst, Massive three-loop Feynman diagrams reducible to $\mathrm{SC}^{*}$ primitives of algebras of the sixth root of unity, Eur. Phys. J. C 8, 311 (1999).

[65] B. A. Kniehl, A. F. Pikelner, and O. L. Veretin, Three-loop massive tadpoles and polylogarithms through weight six, J. High Energy Phys. 08 (2017) 024.

[66] Y. Schröder and A. Vuorinen, High-precision epsilon expansions of single-mass-scale four-loop vacuum bubbles, J. High Energy Phys. 06 (2005) 051.

[67] T. Luthe, Fully massive vacuum integrals at 5 loops, Ph.D. thesis, Bielefeld University, 2015.
[68] T. Luthe, A. Maier, P. Marquard, and Y. Schroder, Complete renormalization of QCD at five loops, J. High Energy Phys. 03 (2017) 020.

[69] A. V. Smirnov and A. V. Petukhov, The number of master integrals is finite, Lett. Math. Phys. 97, 37 (2011).

[70] T. Peraro, Scattering amplitudes over finite fields and multivariate functional reconstruction, J. High Energy Phys. 12 (2016) 030.

[71] A. I. Davydychev, A simple formula for reducing Feynman diagrams to scalar integrals, Phys. Lett. B 263, 107 (1991).

[72] O. V. Tarasov, Connection between Feynman integrals having different values of the spacetime dimension, Phys. Rev. D 54, 6479 (1996).

[73] O. V. Tarasov, Generalized recurrence relations for two loop propagator integrals with arbitrary masses, Nucl. Phys. B502, 455 (1997).

[74] R. N. Lee, spacetime dimensionality D as complex variable: Calculating loop integrals using dimensional recurrence relation and analytical properties with respect to D, Nucl. Phys. B830, 474 (2010).

[75] G. Duplancic and B. Nizic, Reduction method for dimensionally regulated one loop $\mathrm{N}$ point Feynman integrals, Eur. Phys. J. C 35, 105 (2004).

[76] A. Georgoudis, K. J. Larsen, and Y. Zhang, Azurite: An algebraic geometry based package for finding bases of loop integrals, Comput. Phys. Commun. 221, 203 (2017).

[77] X. Liu and Y.-Q. Ma (to be published).

[78] B. Mistlberger, Higgs boson production at hadron colliders at $\mathrm{N}^{3}$ LO in QCD, J. High Energy Phys. 05 (2018) 028.

[79] D. A. Kosower, Direct solution of integration-by-parts systems, Phys. Rev. D 98, 025008 (2018).

[80] S. Borowka, T. Gehrmann, and D. Hulme, Systematic approximation of multiscale Feynman integrals, J. High Energy Phys. 08 (2018) 111.

[81] J. Bohm, A. Georgoudis, K. J. Larsen, H. Schonemann, and Y. Zhang, Complete integration-by-parts reductions of the nonplanar hexagon-box via module intersections, J. High Energy Phys. 09 (2018) 024.

[82] H. A. Chawdhry, M. A. Lim, and A. Mitov, Two-loop fivepoint massless QCD amplitudes within the IBP approach, arXiv:1805.09182.

[83] S. Di Vita, S. Laporta, P. Mastrolia, A. Primo, and U. Schubert, Master integrals for the NNLO virtual corrections to $e$ scattering in QED: The nonplanar graphs, J. High Energy Phys. 09 (2018) 016.

[84] S. Abreu, B. Page, and M. Zeng, Differential equations from unitarity cuts: Nonplanar hexa-box integrals, J. High Energy Phys. 01 (2019) 006.

[85] P. Banerjee, S. Borowka, P. K. Dhani, T. Gehrmann, and V. Ravindran, Two-loop massless QCD corrections to the $g+g \rightarrow H+H$ four-point amplitude, J. High Energy Phys. 11 (2018) 130.

[86] D. Chicherin, T. Gehrmann, J. M. Henn, N. A. Lo Presti, V. Mitev, and P. Wasser, Analytic result for the nonplanar hexabox integrals, J. High Energy Phys. 03 (2019) 042.

[87] P. Mastrolia and S. Mizera, Feynman integrals and intersection theory, J. High Energy Phys. 02 (2019) 139.

[88] X. Xu and L. L. Yang, Towards a new approximation for pair-production and associated-production of the Higgs boson, J. High Energy Phys. 01 (2019) 211. 
[89] J. Davies, G. Mishima, M. Steinhauser, and D. Wellmann, Double Higgs boson production at NLO in the high-energy limit: complete analytic results, J. High Energy Phys. 01 (2019) 176.

[90] S. Badger, C. Brønnum-Hansen, H. B. Hartanto, and T. Peraro, Analytic helicity amplitudes for two-loop five-gluon scattering: the single-minus case, J. High Energy Phys. 01 (2019) 186.

[91] M. K. Mandal and X. Zhao, Evaluating multiloop Feynman integrals numerically through differential equations, arXiv: 1812.03060.
[92] G. Mishima, High-energy expansion of two-loop massive four-point diagrams, J. High Energy Phys. 02 (2019) 080.

[93] A. Kardos, A new reduction strategy for special negative sectors of planar two-loop integrals without Laporta algorithm, arXiv:1812.05622.

[94] H. Frellesvig, F. Gasparotto, S. Laporta, M. K. Mandal, P. Mastrolia, L. Mattiazzi, and S. Mizera, Decomposition of Feynman integrals on the maximal cut by intersection numbers, arXiv:1901.11510. 